\title{
HFSS Design of Hexagonal Monopole Antenna with FR4 Epoxy substrate - 7.34 GHz Ultra-Wide Band Antenna for Smart Shopping Application
}

\author{
Durgesh Chaudhary, Akhilesh Kumar Mishra, FIE
}

\begin{abstract}
The requirement to serve more users and provide more data at more incredible data speeds drives demand for wireless wideband communications. The Ultra-Wide Band (UWB) technology uses nanosecondlong pulses to span an extensive frequency range. The high bandwidth, low power consumption, and less fading due to multipath are attained in UWB communication technologies. This paper explains a hexagonal monopole antenna design using a high-frequency structure simulator (HFSS) and is further optimized for $\mathbf{c}$ better bandwidth. A hexagonal monopole antenna is designed using an FR-4 epoxy metamaterial substrate. The optimum hexagonal bandwidth is attained at a span of 6.13 to $7.34 \mathrm{GHz}$ frequency, and the polar gain is $1.2 \mathrm{~dB}$. This paper provides the UWB antenna design; an optimized bandwidth is achieved as expected, the applications of smart marketing and a discussion of all relevant literature are carried out.
\end{abstract}

Index Terms; High-Performance Antenna; Hexagonal Antenna; Monopole Antenna; HFSS; UWB

\section{INTRODUCTION}

The designated UWB range of frequencies is from $3.1 \mathrm{GHz}$ to $10.6 \mathrm{GHz}$ [1]. Design considerations for this new communications standard received a lot of attention afterward. UWB systems need low-cost antennas with omnidirectional radiation patterns, large bandwidth, and non-dispersive behavior. [2]. Because of these limits, UWB antenna design is more complicated. An antenna is an essential component of wireless communication [3]. Recent antenna designs have all been built with physical features of the output in mind. The Antenna is vital due to the current communication system needs in portable devices [4]. Many scientists are still working on the best ways to minimize the size and weight of multi-band antennas while maintaining acceptable performance [5], [6], [7].

\footnotetext{
Manuscript revised on August 30, 2021 and published on September 10, 2021

Durgesh Chaudhary, Shri Jagdishprasad Jhabarmal Tibrewala University, Jhunjhunu, Rajasthan, India. Email id: durgeshchaudhary8@gmail.com

Akhilesh Kumar Mishra, Shri Jagdishprasad Jhabarmal Tibrewala University, Jhunjhunu, Rajasthan, India.

Email id: akhileshmishra1965@gmail.com
}

Because of its lightweight, compact size, ease of manufacturing, and low cost of implementation, an integrated antenna is among the ones that are being favored for a variety of practical applications. They are essential for different microwave applications, as well as in wireless communication. One side of a dielectric substrate can be used to create basic microstrip antennas [8], [9], [10].

A plane runs parallel to the ground on the other side of the substrate [11]. The patch is usually composed of a conductive material such as copper or gold and comes in various forms, including rectangular, round, triangular, elliptical, and other configurations [12]. Rectangular and circular patch antennas are the most often utilized microstrip patches in practical applications [13]. Integrated antennas are commonly used in wireless communication due to their compact size, low cost, conformability, and convenience of integration with communication devices [14]. The printed wide-slot Antenna's impedance bandwidth is regulated by connecting the tuning stub and the slot. A technique that causes many resonances are used; the frequency widens if the tuning stub is set closer to the ground [15]. Their forms have been described as fork-like, circular, elliptical rectangles, and inverted cones. [16]. Fractal technology's self-similarity is utilized in this project. [17] To create a multi-frequency antenna architecture that meets the FCC standard mask [18].

\section{BACKGROUND}

Many technologies exist to improve the flow of radio communications. The UWB systems can be found in several wireless solutions. [19]. UWB's features are examined via the time domain or frequency domain. Both domains utilize comprehensive radiation information. Usually, differentiation, dispersion, radiation, and losses are the impulse given to the UWB antenna [20]. UWB antenna analysis includes measurement of the peak, breadth, and rings and gain, frequency domain gain, group delay, and mean group delay [21]. In advanced communication technologies like wireless sensor networks, UWB plays a key role with minimum cost and complexity [22]. The authors Amrollah Amini, HomayoonOraizi, and Mohammad Amin Chaychizadeh, suggested a log-periodic square fractal geometry to construct a UWB [23] miniaturized patch antenna (3.1$10.6 \mathrm{GHz}$ ). A $23 \%$ downsizing factor is obtained with a consistent and steady gain in the required band [24]. The radiation pattern is wide, finding excellent uses in UWB radars and medical imaging. Furthermore, the suggested Antenna's time-domain performance is examined. 


\section{E-ISSN: 2321-9637 \\ Available online at www.ijrat.org}

Researchers Amjad Omar, Raed Shubair, explored a UWB coplanar waveguide-fed-coplanar strips square-spiral Antenna operating in a 4-10 GHz frequency band [25]. This antenna design is simple and achieves a $50 \%$ size reduction over other available UWB spiral antennas. A matching resistor is used to generate more significant than $10 \mathrm{~dB}$ of over-the-operational frequency return. The Antenna delivers $6.6-7 \mathrm{GHz}$ circular polarization rangeboresight. Once the design is complete, optimizing the frequency range will be possible. [26]. Discussed were the design consideration, characteristics, and different types of UWB antenna and their application, and performance. Based on literature survey difficulties in UWB technology, intensive study was required. Different antenna structures were created for UWB applications, resulting in antenna design as an attractive study topic [27], [28].

\section{UWB FOR SHOPPING}

Retailers and customers alike benefit from UWB's improved shopping experiences and closer relationships. Everyone saves time with automatic and instant payments. Unlocking safe mobile transactions with UWB and saving time at the register. Simply walk up to the cashier, confirm the purchase, and leave [29]. The smartphone also acts as a key to an unmanned store, with UWB verifying the identity and allowing purchases [30]. Toll plazas, petrol stations, and parking garages can accept UWB as a payment card. Analytics that indicate how many consumers visit a business, how long they stay in a specific location, or detect shopping trends help retailers better understand what works and what doesn't work [31]. When customers go past a display or a kiosk, they can view personalized advertising based on data from their mobile devices. An indoor guide to conference rooms and other meeting venues can help visitors locate their planned sessions during conferences, tradeshows, exhibits, and other large-scale events [32]. Foot traffic in a closed environment can be estimated [33]. UWB also pushes the trend of drone-controlled deliveries to desired place [34].

\section{METHODS}

The top and bottom open-circuit walls and the patches mimic a resonant cavity. Only specified modes of resonance frequencies may occur in a cavity. When the Antenna resonates, it produces a strong field in the cavity and a strong current on the surface of the patch. It produces a lot of antenna radiation. [35]. Thin substrate approximation can be written as,

$$
E_{t}=0 \text { and } E=z E_{z}
$$

Since the substrate is so thin, the electric field vector is nearly isotropic, and therefore represented as:

$$
E(x, y, z,) \approx z E_{z}(x, y)
$$

The patch cavity of the magnetic field is given by

$$
H=-\frac{1}{j \omega \mu} \nabla \mathrm{XE}
$$

$$
\begin{aligned}
& =-\frac{1}{j \omega \mu} \nabla X\left(z E_{z}(x, y)\right) \\
& =-\frac{1}{j \omega \mu}\left(-z X \nabla E_{z}(x, y)\right) \\
H(x, y) & =\frac{1}{j \omega \mu}\left(z X \nabla E_{z}(x, y)\right)
\end{aligned}
$$

Figures show the proposed Antenna's geometry with its parameters. An antenna substrate of $1.6 \mathrm{~mm}$ thickness and 4.4 relative permittivities is easy to work with, resulting in a cheap and fast antenna. Microstrip antennas require three design criteria:

A. The Antenna's resonance frequency (fr) must be chosen correctly. An antenna built for a UWB communication system should work between 3.1 to $10.6 \mathrm{GHz}$. The resonance frequency for design is 7.34 $\mathrm{GHz}$.

B. The dielectric constant of 4.4 for FR4 epoxy. The Antenna is of smaller size because of a high dielectric constant substrate.

C. Microstrip Patch antenna size (diameter) For wireless applications, the Antenna should not be bulky. The dielectric substrate is adjusted at $1.6 \mathrm{~mm}$. The critical design parameters include:

$$
\begin{gathered}
f_{0}=7.34 \mathrm{GHz} \\
\varepsilon_{r}=4.4 \\
h=1.6
\end{gathered}
$$

The equation (3) produces the effective dielectric constant $\epsilon_{\text {eff }}=\frac{\varepsilon_{r}+1}{2}(1+0.3 * h)$

The equation (4) for calculating the Microstrip antenna's length is given as

$$
L_{s}=\frac{0.4 * c}{f_{r} * \sqrt{\varepsilon_{e f f}}}
$$

Calculation of ground plane width $(\mathrm{Wg})$ : ground plane width may be determined using equation (5)

$$
W_{g}=\frac{1.3 * c}{f_{r} * \sqrt{\varepsilon_{e f f}}}
$$

Calculation of ground-level ( $\mathrm{Lg})$ : The ground plane length is found via equation (6)

$$
L_{g}=\frac{0.3 * c}{f_{r} * \sqrt{\varepsilon_{e f f}}}
$$

Resonant frequency calculation (fr): resonant frequency (fr) equation (7), 


$$
f_{r} 3+\frac{2}{\sqrt{\varepsilon_{e f f}}}\left[\frac{21}{L_{s}}+\frac{65}{W_{g}}+\frac{18}{L_{g}}-3\right]
$$

The dimensions of the strip monopole antenna are $a=10$ $\mathrm{mm}$ for hexagonal and

$\mathrm{Lg}=20 \mathrm{~mm}, \mathrm{Wg}=45 \mathrm{~mm}, \mathrm{H}=9 \mathrm{~mm}, \mathrm{D}=3 \mathrm{~mm}, \mathrm{~A}=1,6 \mathrm{~mm}$, and finally the $\mathrm{Gr}=4,44$.

\section{DESIGN}

Figures show the suggested Antenna's shape as well as its parameter. The antenna design calls for a conventional FR4 epoxy substrate with a thickness of $1.6 \mathrm{~mm}$ and 4.4 of primitivity, making it economical and uncomplicated to construct. The following are the three most important factors to consider while designing microstrip antennas: Frequency of resonance (fr): The Antenna's resonance frequency should be chosen carefully. The frequency range of UWB communication systems is $3.1 \mathrm{GHz}$ to $10.6 \mathrm{GHz}$. Thus, the Antenna must be capable of operating in this range. The $5.5 \mathrm{GHz}$ resonant frequency was chosen for the design. The substrate's dielectric constant $(€ \mathrm{r})$ is: FR4 epoxy, consisting of a 4.4 dielectric constant, was chosen as the dielectric material for our design. Because it minimizes the Antenna's size, a substrate with a high dielectric constant was used.

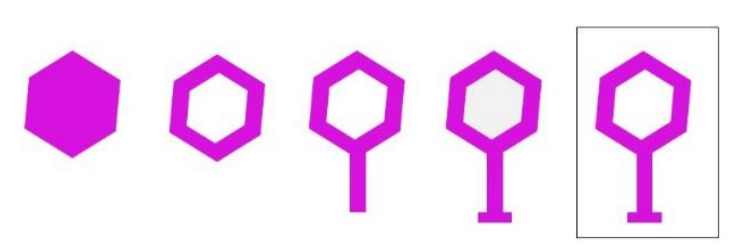

Figure 1: Geometry of Hexagonal Antenna

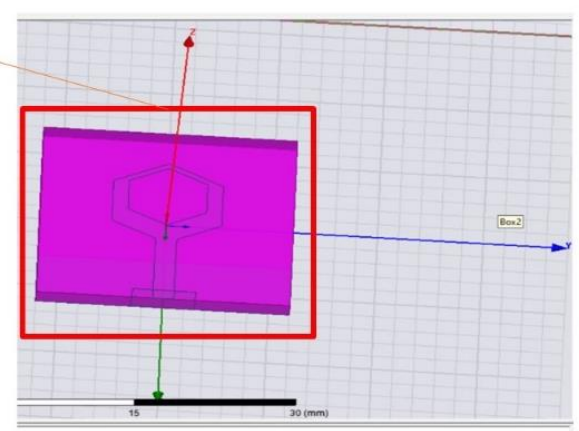

(a) Hexagonal Antenna Design

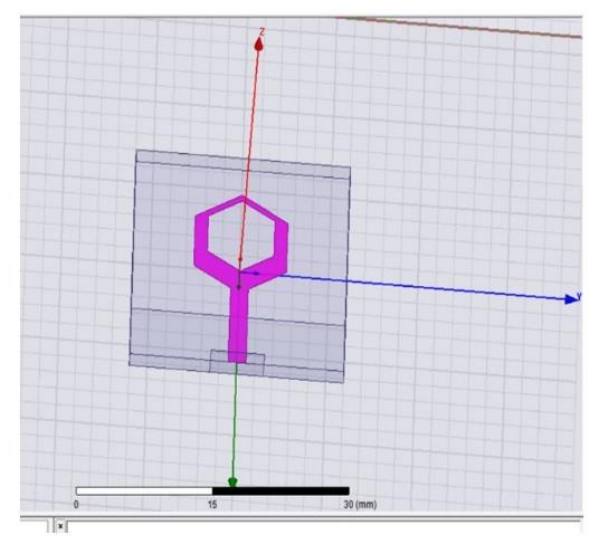

Figure 2: (b) Hexagonal Antenna with FR4- Epoxy Substrate

The drawing depicts the geometry of the proposed Coplanar Waveguide (CPW) fed dual-band hexagonal monopole antenna. The Antenna's dielectric constant was 4.4, and its thickness was $1.6 \mathrm{~mm}$; hexagonal antenna prototypes are created, built, and tested. HFSS was used to simulate the proposed Antenna. 


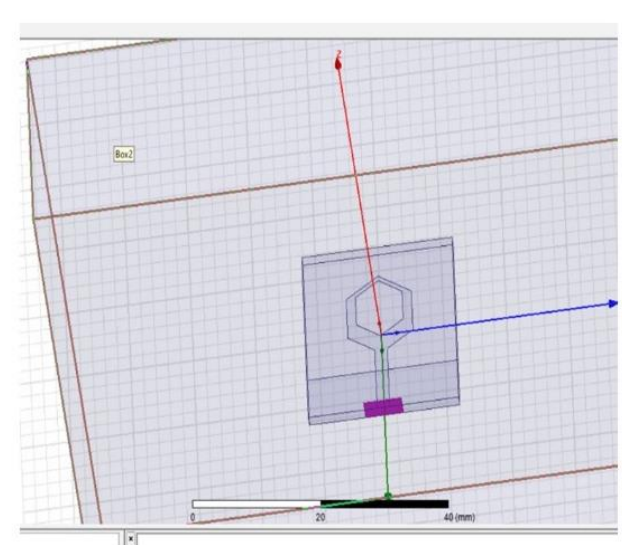

(a) Wave Port Antenna Connector

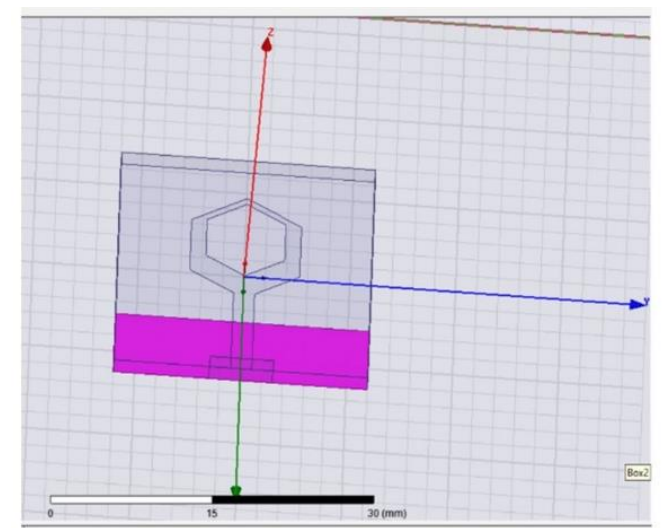

Figure 3: (b) Rectangular base in the ground of the Antenna

\section{Optimization}

HFSS was used to investigate the performance of hexagonal shape antennas. Figures 6, 7, and 8 depict the simulated return loss, Voltage Standing Wave Ratio (VSWR), and the measured return loss; VSWR and Figure 8 depict the strip monopoly antenna radiation pattern from $1.34 \mathrm{GHz}$ to $5.65 \mathrm{GHz}$.

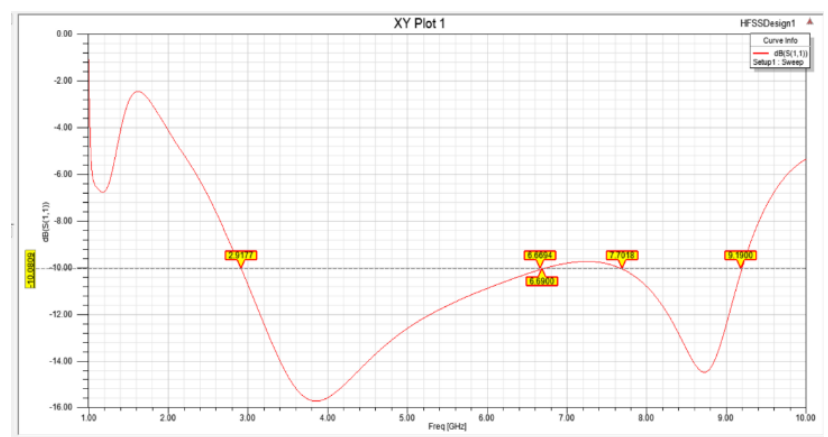

Figure 4: Loss Curve for Hexagonal Antenna using HFSS Before optimization

Dual-band, 2.917-6.66, 6.69-7.70

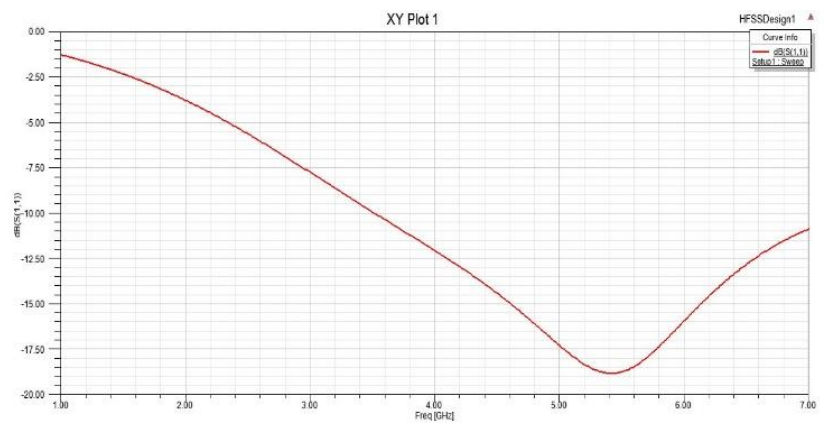

Figure 5: VSWR Curve for Hexagonal Monopole Antenna using HFSS

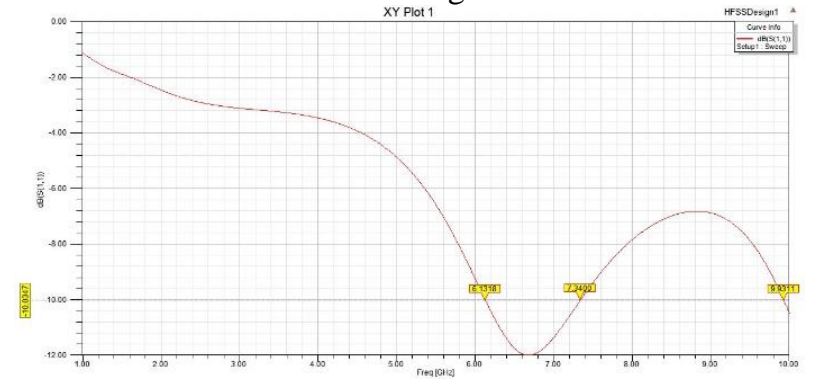

Figure 6: Loss Curve Hexagonal Monopole Antenna after Optimization using HFSS

Position of ground: 2.5, -12, 0, X size: 10, Bandwidth: 6.13-7.34
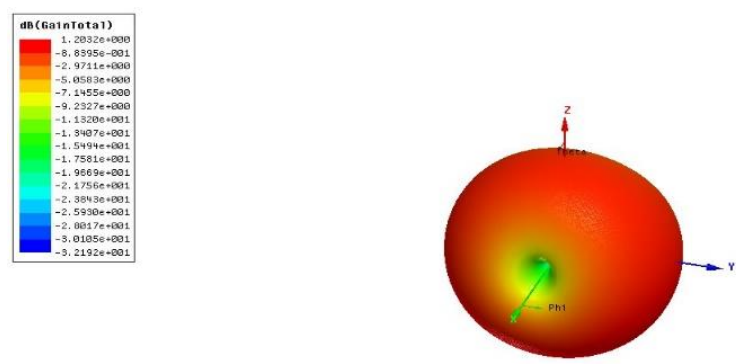

Figure 7: Radiation Pattern Measured from 6.13 to 7.34 $\mathrm{GHz}$ using HFSS
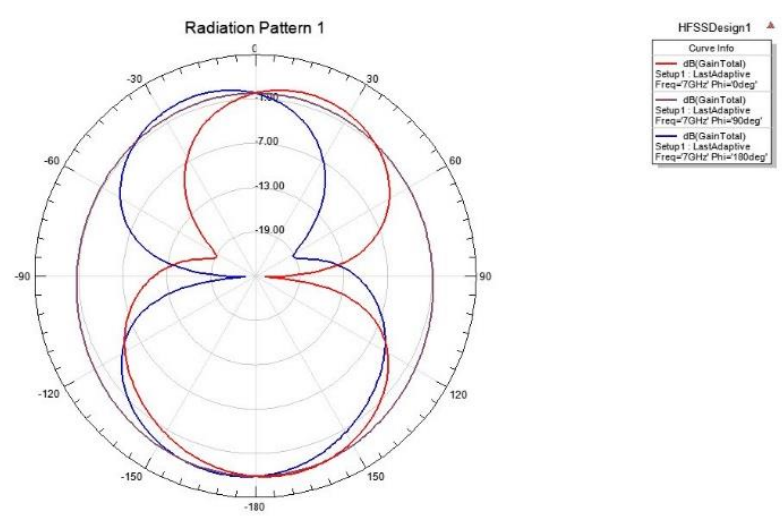

Figure 8: Radiation Pattern for 7.34 GHz using HFSS VII. CONCLUSION 


\section{International Journal of Research in Advent Technology, Vol.9, No.8, August 2021 E-ISSN : 2321-9637 \\ Available online at www.ijrat.org}

The design and research are primarily helpful for operating an Antenna at a specified frequency band. The hexagonal Antenna was designed and simulated with Ansoft HFSS, and the practical results were obtained by evaluating the constructed antennas with a vector network analyzer. The hexagonal strip monopoly has a frequency of resonance of $7.34 \mathrm{GHz}$, and UWB impedance bandwidth $(-12 \mathrm{~dB})$ ranges from 6.13 to $9.93 \mathrm{GHz}$. The observed simulation findings, where it is observed at $6.13 \mathrm{GHz}$ to $7.34 \mathrm{GHz}$ as erroneous results. Simulated results are seen while actual results are observed in increments of dual-band 2.917-6.66 $\mathrm{GHz}, 6.69-7.70 \mathrm{GHz}$. The hexagonal range of frequency is $7.34 \mathrm{GHz}$. The suggested antennas provide positive gain and efficiency with almost Omni-directional radiation characteristics appropriate for next-generation UWB applications. UWB is expected to be revolutionized the shopping experience. In the wake of the global pandemic of covid-19, social distancing is imperative to protect from the spread of the virus. Automatic checkout systems developed with the integration of UWB are the best solutions. The paper's future scope is to conduct a comparative analysis between other frequency ranges to estimate the frequency and gain for the Hexagonal Monopole antenna.

\section{REFERENCES}

[1] J. Liang, "Antenna study and design for ultra-wideband communication applications," Doctoral dissertation, University of London, 2006.

[2] A. KHINE, Z. LWIN, K. S. Lwin and Z. NAING, "Microstrip Bandpass Filter for Ultra-Wideband Direct-Conversion Receiver (6-10.6 GHz)," 2014.

[3] H. Schantz, "Ultrawideband antennas," Artech House, Inc, 2005.

[4] S. Ghosh and D. Sen, "An inclusive survey on array antenna design for millimeter-wave communications," IEEE Access, vol. 7, pp. 83137-83161, 2019.

[5] M. S. Sharawi, "Printed multi-band MIMO antenna systems and their performance metrics [wireless corner].," IEEE Antennas and Propagation Magazine, vol. 55, no. 5, pp. 218-232, 2013.

[6] S. M. Ali, C. Sovuthy and M. A. Imran, "Recent advances of wearable antennas in materials, fabrication methods, designs, and their applications: State-of-the-art," Micromachines, vol. 11, no. 10 , p. $888,2020$.

[7] P. Marichamy, "Compact slot based triangular multi-band microstrip patch antenna for C and X band applications," 2021.

[8] S. Dhar, R. Ghatak, B. Gupta and D. R. Poddar, "(2013). A wideband Minkowski fractal dielectric resonator antenna.," IEEE transactions on antennas and propagation, vol. 61, no. 6, pp. 2895 2903, 2013.

[9] A. Y. Ashyap, Z. Z. Abidin, S. H. Dahlan, and et. al., "Inverted Eshaped wearable textile antenna for medical applications.," IEEE Access, vol. 6, pp. 35214-35222, 2018.

[10] R. Das and H. Yoo, "A multi-band antenna associating wireless monitoring and nonleaky wireless power transfer system for biomedical implants," IEEE Transactions on Microwave Theory and Techniques, vol. 65, no. 7, pp. 2485-2495, 2017.

[11] G. Zheng, A. A. Kishk, A. W. Glisson and Yakovle, "(2004). Simplified feed for modified printed Yagi antenna.," Electronics Letters, vol. 40, no. 8, pp. 464-466, 2004.

[12] C. Hannachi and S. O. Tatu, "Performance comparison of $60 \mathrm{GHz}$ printed patch antennas with different geometrical shapes using miniature hybrid microwave integrated circuits technology," IET Microwaves, Antennas \& Propagation, vol. 11, no. 1, pp. 106-112, 2017.

[13] I. Singh and V. S. Tripathi, "Microstrip patch antenna and its applications: a survey," Int. J. Comp. Tech. Appl, vol. 2, no. 5, pp. 1595-1599, 2011

[14] A. S. Ghattas and E. M. Khaled, "A compact ultra-wideband microstrip patch antenna designed for $\mathrm{Ku} / \mathrm{K}$ bands applications.," 2017 Japan-Africa Conference on Electronics, Communications and Computers (JAC-ECC) IEEE, pp. 61-64, 2017.

[15] B. Mohamadzade, R. M. Hashmi and R. B. Simorangkir, "Recent advances in fabrication methods for flexible antennas in wearable devices: State of the art," Sensors, vol. 19, no. 10, p. 2312, 2019.

[16] D. D. Krishna, M. Gopikrishna and C. K. Aanandan, "Ultrawideband slot antenna with band-notch characteristics for wireless USB dongle applications," Microwave and Optical Technology Letters, vol. 51, no. 6, pp. 1500-1504, 2009.

[17] S. V. Yashwant, B. D. Govind, and R. S. Dhanraj, "PRINTED WIDE-SLOT ANTENNA FOR WLAN APPLICATION," IERjournals, 2018.

[18] G. W. Hein, "Status, perspectives, and trends of satellite navigation," Satellite Navigation, vol. 1, no. 1, pp. 1-12, 2020.

[19] N. Sasireka and M. R. Devi, "A literature survey on ultra-wideband antennas," IJIRST-International Journal for Innovative Research in Science \& Technology, vol. 3, no. 09, pp. 17-22., 2017.

[20] G. Madanan and D. D. Krishna, "Time Domain Performance Evaluation of UWB Antennas," UWB Technology IntechOpen, 2020.

[21] H. H. Sun, Y. H. Lee, W. Luo, L. F. Ow and Yusof, "Compact Dual-Polarized Vivaldi Antenna with High Gain and High Polarization Purity for GPR Applications," Sensors, vol. 21, no. 3 , p. 503, 2021.

[22] A. Coluccia and A. Fascista, "A review of advanced localization techniques for crowdsensing wireless sensor networks," Sensors, vol. 19, no. 5, p. 988, 2019.

[23] I. Elfergani, A. S. Hussaini and J. Rodriguez, "Fundamentals of Antenna Design, Technologies and Applications," Antenna Fundamentals for Legacy Mobile Applications and Beyond. Springer, Cham., pp. 3-36, 2018.

[24] C. E. Guan and T. Fujimoto, "Design of a Wideband L-Shape Fed Microstrip Patch Antenna Backed by Conductor Plane for Medical Body Area Network," Electronics, vol. 9, no. 1, p. 21, 2020.

[25] A. Omar and R. Shubair, "UWB coplanar waveguide-fed-coplanar strips spiral antenna," 2016 10th European Conference on Antennas and Propagation (EuCAP), IEEE, pp. 1-2, 2016.

[26] S. Koziel and A. Pietrenko-Dabrowska, "Rapid multi-objective optimization of antennas using nested kriging surrogates and single-fidelity EM simulation models," Engineering Computations, 2019.

[27] H. Banting, "Novel Structures and Materials for Broadband Antennas," Doctoral dissertation, Queen's University, Canada, 2020.

[28] W. Su, J. Zhu, H. Liao, and M. M. Tentzeris, "Wearable antennas for cross-body communication and human activity recognition," IEEE Access, vol. 8, pp. 58575-58584, 2020.

[29] M. Contigiani, R. Pietrini, A. Mancini, and Zinga, "Implementation of a tracking system based on UWB technology in a retail environment," 2th IEEE/ASME International Conference on Mechatronic and Embedded Systems and Applications, pp. 1-6, 2016.

[30] T. M. Fernández-Caramés and et. al., "Towards an autonomous industry 4.0 warehouse: A UAV and blockchain-based system for inventory and traceability applications in big data-driven supply chain management," Sensors, vol. 19, no. 10, p. 2394, 2019.

[31] T. Zhou, M. Xiao, Y. Liu, Y. Cheng, and Y. Liu, "Research on Indoor UWB Positioning Based on Expectation Maximization in 
International Journal of Research in Advent Technology, Vol.9, No.8, August 2021

E-ISSN : 2321-9637

Available online at www.ijrat.org

NLOS Environment," Concurrency and Computation: Practice and Experience, p. e6278, 2021.

[32] X. Quan, D. H. Yim, and S. H. Cho, "Shopping store management system based on IR-UWB radar sensors," 2016 IEEE International Conference on Network Infrastructure and Digital Content (ICNIDC), pp. 481-485, 2016.

[33] M. De Sanctis, A. Conte, T. Rossi, and Di Domenico, "CIR-Based Device-Free People Counting via UWB Signals," Sensors, vol. 21, no. 9, p. 3296, 2021.

[34] J. Tiemann and C. Wietfeld, "Scalable and precise multi-UAV indoor navigation using TDOA-based UWB localization," International conference on indoor positioning and indoor navigation (IPIN), pp. 1-7, 2017.

[35] S. Mercader-Pellicer, W. Tang and D. L. Bresciani, "Angularly Stable Linear-to-Circular Polarizing Reflectors for Multiple Beam Antennas," IEEE Transactions on Antennas and Propagation, 2021. 